\title{
Appendix Mixed Adenoneuroendocrine Carcinoma
}

National Cancer Institute

\section{Source}

National Cancer Institute. Appendix Mixed Adenoneuroendocrine Carcinoma. NCI

Thesaurus. Code 143564.

A carcinoma that arises from the appendix. It is characterized by the presence of a malignant glandular epithelial component and a malignant neuroendocrine component. At least 30 percent of either component should be present for the diagnosis to be made. 